\title{
ON RISK MODEL WITH DIVIDENDS PAYMENTS PERTURBED BY A BROWNIAN MOTION - AN ALGORITHMIC APPROACH
}

\author{
BY
}

\author{
ESTHER FROSTIG
}

\begin{abstract}
Assume that an insurance company pays dividends to its shareholders whenever the surplus process is above a given threshold. In this paper we study the expected amount of dividends paid, and the expected time to ruin in the compound Poisson risk process perturbed by a Brownian motion. Two models are considered: In the first one the insurance company pays whatever amount exceeds a given level $b$ as dividends to its shareholders. In the second model, the company starts to pay dividends at a given rate, smaller than the premium rate, whenever the surplus up-crosses the level $b$. The dividends are paid until the surplus down-crosses the level $a, a<b$. We assume that the claim sizes are phase-type distributed. In the analysis we apply the multidimensional Wald martingale, and the multidimensional Asmussesn and Kella martingale.
\end{abstract}

\section{KEYWORDS}

Wald martingale, stopping time, optional sampling theorem, Kella and Whitt martingale.

\section{INTRODUCTION}

De Finetti (1957) was the first to suggest the reduction of the free reserves by paying dividends to the shareholders when the reserve reaches a high level greater than the initial reserve. For the discrete time Binomial model, De Finetti found that the optimal strategy is the barrier strategy, that is, dividends are paid whenever the surplus process reaches a fixed high level greater than the initial reserve.

Since De Finetti's (1957) paper, the reserve process with dividends has been studied by many researchers.

In this paper we consider two models:

Model I. When the surplus reaches the level $b$, all the surplus overflow above $b$ is paid as dividends. 
Model II. Whenever the surplus reaches the level $b$, dividends are paid at rate $d<c$, where $c$ is the premium rate. Dividends are paid until the risk process reaches the level $a, a<b$, and are paid, again, in the same pattern when the surplus reaches the level $b$ again.

Many works studied Model I. Most of them assumed that the claims arrive according to a Poisson process.

Irbäck (2003) obtained numerous results concerning the time to ruin when the barrier is asymptotically infinity. He assumed that the initial reserve is either close to the barrier, or very small compared to the barrier.

In most of the papers an integro-differential equation is derived to obtain the expected dividends paid, and the expected time to ruin. Bühlmann (1970) discussed various dividend policies in discrete time. For the continuous time he presented an integro-differential equation for the expected discounted dividends paid until ruin.

Gerber (1979) also discussed an integro-differential equation for the cumulative expected discounted dividend payout. By presenting a certain martingale he obtained an expression for the Laplace transform of the time to ruin.

Paulsen and Gjessing (1997) considered a risk process with a stochastic return on the investment, where both the risk process and the return process are Lévy processes with a compound Poisson component, and a Brownian motion component. They obtained an integro-differential equation for the expected discounted dividends.

In 1998 Gerber and Shiu presented their famous paper where they defined the penalty function to analyze the discounted time to ruin. Gerber and Shiu (1998), Section 7, presented an integro-differential equation for the discounted dividends. Some researchers applied Gerber and Shiu's penalty function to study the dividend problem. Lin et al. (2003) obtained an integral equation for the expected penalty function. From this equation they derived expressions for the Laplace transform of the time to ruin, and expressions for the moment of the deficit at ruin. Both quantities are given as functions of compound geometric distribution. Applying similar methods, Dickson and Waters (2004) found an integro-differential equation for the discounted moments of the dividend payments for the classical compound Poisson risk process. They also analyzed a discrete model and showed how the discrete time model can approximate the continuous one.

Zhou (2005) considered Poisson arrival process and implied results from spectrally negative Lévy process to obtain the joint distribution of the surplus before and after ruin. The results are given as a function which is a solution to an integral equation. In a more recent paper, Gerber and Shiu (2006) discussed the optimal dividend rate in the case of Poisson arrival process. They obtained an intero-differential equation for the expected dividends in the case of constant dividend barrier.

$\mathrm{Li}$ and Garrido (2004) and Albrecher et al. (2005), assumed Erlang interarrival times. Li and Garrido extended Lin et al.'s (2003) results, and Albrecher 
et al. (2005) obtained an integro-differential equations for the moment generating function and for the moments of the dividends.

Gereber and Shiu (2004) considered the case where surplus process is modeled as Brownian motion. They found the expected discounted dividends, the moment generating function of the dividends, and the Laplace transform of the time to ruin. Frostig (2005a) considered the classical model with a constant dividends barrier with phase type distributed claim sizes. In this case she obtained an algorithm to calculate the expected dividends and the expected time to ruin.

In a recent paper $\mathrm{Li}$ (2006) obtained an integro-differential equation for the $n$-th moment of the discounted dividends in the case of the classical compound Poisson model perturbed by Brownian motion. Li (2006) obtained the expected dividends paid as integrals of the non-ruin probabilities in a classical compound Poisson risk model perturbed by diffusion.

Lately, a few papers have considered Model II. Lin and Pavlova (2006) considered the classical compound Poisson risk model, where dividends are paid at a smaller rate than the premium rate whenever the surplus is above the barrier. They obtained an integro-differential equation for Gerber-Shiu penalty function, and derived the ruin probabilities as functions of the ruin probability in the classical model without dividends. Gerber and Shiu (2006) obtained an integro-differential equation for the expected discounted dividends, and moment generating function for the time to ruin. In a recent paper Bratiychunk and Derfla (2007) exploited the properties of Lèvy process without negative jumps, to find the ruin probability. The ruin probability was found as a function of a solution to an integral equation which is difficult to find explicitly.

In all the papers explicit expressions were obtained for the case of Erlang or mixture of Erlang distributions claims amount.

Frostig (2005b) assumed phase type claim size distribution. By exploiting Kella and Whitt's (1992) martingale and Asmussen and Kella's (2000) martingale, she obtained algorithm to derive numerically the expected time to ruin, and the expected dividend payments.

In this paper we consider Models I and II with Poisson claim arrivals that is perturbed by diffusion. The purpose is to obtain algorithms that are easy to implement to calculate the expected dividends, expected time to ruin, and the distribution of the deficit at ruin. For the second model we also obtain the ruin probability. We assume that the claims are phase-type distributed. The family of the phase type distributions is dense in the set of all distributions. This means that every distribution can be approximated by phase type distribution. The special structure of the phase type distributions has been applied in many applied probability models, especially in queueing theory (Neuts (1981), Asmussen (2003)), to derive quantities of interest. It was also applied in risk theory to obtain explicit formula for the ruin probabilities. (See Asmussen (2000) and references therein).

In Section 2 we analyze the first model. We obtain the distribution of the dividends and the distribution of the deficit at ruin. We derive the expected time 
to ruin. The second model is presented in Section 3. For the case where the ruin probability is 1 , we obtain the expected dividends, the expected time to ruin, and the distribution of the deficit. We also derive the ruin probability when it is less than 1 .

\section{Model I}

\subsection{Model description and general result}

Consider the risk process $\{R(u, t), t \geq 0\}$, where

$$
R(u, t)=u+c t-\sum_{j=1}^{N(t)} W_{j}+\sigma B(t) .
$$

Claims arrive according to a Poisson process $N(t)$ with rate $\lambda>0, W_{i}$ is the $i$ th claim amount, $W_{i}$ are i.i.d distributed as a random variable $W$, the premium income rate is $c>0$, and the initial reserve is $u$. The risk process is perturbed by a Brownian motion $B(t)$ with volatility coefficient $\sigma>0$. We assume that the claims amount, the arrival process, and the Brownian motion, are independent.

Let $b>u$. In this section we consider model I, where all the surplus overflow above $b$ is paid as dividends.

Let

$$
\begin{aligned}
X(u, t) & =b-R(u, t)=b-u+X(t), \text { where } \\
X(t) & =\sum_{i=1}^{N(t)} W-c t+\sigma B(t) .
\end{aligned}
$$

Note that $X(u, 0)=b-u$. The process $X(t)$ is a Lévy process with no negative jumps, and with a Lévy exponent $\psi(\theta)$, where

$$
\psi(\theta)=\log \left(\mathbb{E}\left[e^{\theta X(1)}\right]\right)=-c \theta+\sigma^{2} \theta^{2} / 2+\lambda\left(\mathbb{E} e^{\theta W}-1\right) .
$$

Let $L(t)=-\inf \{X(s): 0 \leq s \leq t\}$, and $L(u, t)=(L(t)-(b-u))^{+}$, where $x^{+}=$ $\max (x, 0)$. Let $V(u, t)=X(u, t)+L(u, t)$. Note that $L(u, t$,$) is the total dividends$ paid up to time $t$ when the initial reserve is $u, u \leq b . L(u, t)$ is called the local time process at 0 for the process $V(u, t)$, (see Asmussen (2003), p. 250-251). For properties of the local time for Lévy processes Bertoin (1996), Chapters IV and V.

Let $U_{b}(u, t)=b-V(u, t)$, be the surplus process in the presence of the constant barrier. The process $U_{b}(u, t)$ describes the modified surplus process when all the surplus above $b$ is paid as dividends. Let $\tau_{b}(u)=\inf \left\{t: U_{b}(u, t) \leq 0\right\}=$ $\inf \{t: V(u, t) \geq b\} . \tau_{b}(u)$ is the time to ruin when the initial reserve is $u \leq b$. Let $D_{b}(u)=L\left(u, \tau_{b}(u)\right)$ be the total dividends paid until ruin occurs, and $\boldsymbol{\ell}_{b}(u)=$ 
$\mathbb{E}\left[D_{b}(u)\right]$. Under the barrier policy, ruin occurs with probability 1 . The objective in this section is to obtain expressions for the expected time to ruin $\mathbb{E}\left[\tau_{b}(u)\right]$, and the expected dividends paid until ruin occurs- $\mathbb{E}\left[D_{b}(u)\right]=\boldsymbol{\ell}_{b}(u)$.

Let $T(u, 0)=\inf \{t: X(u, t) \leq 0\}$ be the first time that the process $X(u, t)$ hits 0 , and $T(u, b)=\inf \{t: X(u, t) \geq b\}$ the first time that the process $X(u, t)$ hits or exceeds $b$. Let $T(u, 0, b)=\min (T(u, 0), T(u, b))$. Note that when $T(u, 0, b)=$ $T(u, b)$, then ruin occurs before dividends are paid.

Let $a>0$. Denote by $T_{a}$ the first passage time of the process $V$ in $[a, \infty), T_{a}=$ $\inf \{t \geq 0: V(t)=a\}$.

Proposition 2.1. $L\left(T_{a}\right)$ is exponentially distributed.

The proof of this proposition appears in Bertoin (1996), p. 144 (See also p. 123, exercise 1).

We are now ready to state the first result. In the following proposition we assume that the claim amounts are i.i.d, with a general distribution $G$.

Proposition 2.2. When $u=b$ the amount of dividends paid until ruin $L\left(b, \tau_{b}(b)\right)$ is exponentially distributed. When $b>u$, the amount of dividends paid until ruin occurs is a mixture of an exponential distribution and a degenerate distribution at 0 .

The case $u=b$ is immediate from Proposition 2.1. Assume that $u<b$. Let $q(u)=$ $P(T(u, 0)<T(u, b))$. With probability $q(u)$, the process $X(u, t)$ reaches 0 before $b$. Once reaching 0 , the amount of dividends paid until ruin is exponentially distributed. With probability $1-q(u), X(u, t)$ reaches the level $b$ before 0 (ruin occurs before dividend payment); thus ruin occurs before dividends are paid. In this case the amount of dividends is zero.

Remark 2.3.

1. Gerber and Shiu (Section 5, (2004)) obtained the same result in the case where the risk process is a Brownian motion with a drift without jumps, while Dickson and Waters (Section 3, (2004)) obtained the same result for the classical risk process (without Brownian motion).

2. $\mathrm{Li}(2006)$ arrived at the same result by deriving the moment generating function of the dividends by applying integro-differential equation.

Remark 2.4. Bertoin ((1996), Theorem 8 of Chapter VII) shows that

$$
q(u)=\frac{Q(u)}{Q(b)}
$$

where $Q:[0, \infty) \longrightarrow[0, \infty)$ is the unique continuous increasing function with Laplace transform

$$
\int_{0}^{\infty} e^{-\alpha x} Q(x) d x=\frac{1}{c(-\alpha)},
$$


where $\psi$ is as per (2.4). See also discussions on the compound Poisson risk process in Zhou (2005, p. 98), and Bartiychung and Derfla (2007, eq. 6).

In the sequel we will find $q(u)$ and the expected dividends explicitly for the case where $G$ is phase type. Thus for this family of distribution we identify explicitly the parameters of the distribution of the dividends. The probability $q(u)$ was obtained by Gerber and Shiu (Section 5, (1996)) when the risk process evolves as a Brownian motion.

\subsection{Phase type distribution}

From here on we assume that the claim size is Phase type distributed with representation $(n, \boldsymbol{\beta}, \mathbf{T})$. A phase-type distribution with representation $\left(n,\left(\beta_{0}, \boldsymbol{\beta}\right), \mathbf{T}\right)$, or in abbreviation $\left(\left(\beta_{0}, \boldsymbol{\beta}\right), \mathbf{T}\right)$, is the distribution to absorption in a Markov jump process with $n$ transient states, and one absorbing state, say state 0 , where absorbing is certain from each transient state. The transition rate matrix among the transient states is denoted by $\mathbf{T}$. The initial probability vector for starting in the transient state is $\boldsymbol{\beta}=\left(\beta_{1}, \cdots, \beta_{n}\right)$. The probability of starting in the absorbing state and thus being immediately absorbed is $\beta_{0}$ - the atom at 0 . Let $\mathbf{t}=-\mathbf{T} \mathbf{1}$, be the vector of the transition rates to the absorbing state. $\mathbf{1}$ denotes a column vector with all components equal to 1 . If $W$ has a phase type distribution then

$$
\begin{aligned}
& \mathbb{E} e^{s W}=\boldsymbol{\beta}(-s \mathbf{I}-\mathbf{T}) \mathbf{t} \\
& \mathbb{E}[W]=-\boldsymbol{\beta} \mathbf{T}^{-1} \mathbf{1}, \text { when the expectations exist. }
\end{aligned}
$$

Here $\mathbf{I}$ is the identity matrix. When there is no atom at 0 , we denote the representation by $(\boldsymbol{\beta}, \mathbf{T})$. Note that the family of phase type distributions includes the exponential distribution, Erlang distributions, hyper-exponential distributions, Coxian distributions. This family is dense in the weak convergence sense in the set of all probability distributions on $(0, \infty)$. Thus every distribution on $(0, \infty)$ can be approximated by phase-type distribution (Asmussen (2003, chapter III, Asmussen (2000)). References for the properties of Phase-type distributions and their applications to queueing theory are Neuts (1981), Asmussen (2003), chapter III. For applications of Phase type distribution in risk theory, see Asmussen (2000), Chapter VII. For statistical procedures that approximate a given distribution function by Phase type distribution, see Asmussen, Nerman and Olsson (1996), Asmussen (2000), and references therein.

\subsection{The fluid description}

In the sequel we apply the fluid description of $X(u, t)$ and $V(u, t)$. Consider the process $X^{f}(u, t)$, which is constructed from the process $X(u, t)$, as follows: Let $T_{i}$ be the time of the $i$ th jump (claim arrival) in the process $X(u, t)$. For 
$0 \leq t \leq T_{1}, X^{f}(u, t)=X(u, t)$. Assume that at time $T_{1}$ there is a jump of size $W_{1}$ in the processes $X(u, t)$. At this time the processes $X^{f}(u, \cdot)$ start to increase linearly at rate 1 for $W_{1}$ time units. Thus $X^{f}\left(u, T_{1}+W_{1}\right)=X\left(u, T_{1}^{+}\right)$, where $X\left(u, T_{j}^{+}\right)$ is the value of the process $X(u, t)$ immediately after the $j$ th jump. Assume that $X^{f}\left(u, T_{n}+\sum_{j=1}^{n} W_{j}\right)=X\left(u, T_{n}^{+}\right)$. Then $X^{f}\left(u, T_{n}+\sum_{j=1}^{n} W_{j}+t\right)=X\left(T_{n}+t\right)$, for $0 \leq t \leq T_{n+1}-T_{n}$. Let $L^{f}(b, t)=-\inf \left\{X^{f}(b, s), 0 \leq s \leq t\right\}$, and for $u<b$ let $L^{f}(u, t)=\left(L^{f}(b, t)-(b-u)\right)^{+}$. Note The description of $X^{f}$ implies that for $u \leq b$, $L^{f}\left(u, t+\sum_{j=1}^{N(t)} W_{j}\right)=L(u, t)$. Let

$$
V^{f}(u, t)=X^{f}(u, t)+L^{f}(u, t) .
$$

Let $\tau^{f}(u)=\min \left\{t: V^{f}(u, t) \geq b\right\}$. The above construction implies that $\tau^{f}(u)=$ $\tau(u)+\sum_{j=1}^{N(\tau)} W_{j}$, and $L(u, \tau(u))=L^{f}\left(u, \tau^{f}(u)\right)$.

Let's consider a jump Markov process $J(t)$ with state space $0,1, \cdots, n$. The sojourn time of the process at state 0 is exponentially distributed with rate $\lambda$. Upon leaving state 0 , the process jumps to state $i$ with probability $\beta_{i}, i=1, \cdots, n$. The sojourn time in state $i, i=1, \cdots, n$ is exponentially distributed at rate $\left(-T_{i, i}\right)$. The process jumps to state $j \neq i, j=1, \cdots, n$ with probability $T_{i, j} /\left(-T_{i, i}\right)$ and with probability $t_{i} /\left(-T_{i, i}\right)$ it jumps to state 0 . The process $X^{f}(u, t)$ behaves as follows: When $J(t)=0, X^{f}(u, t)$ behaves as a Brownian motion with a drift $-c$, thus with a Lévy exponent $\psi_{0}(\alpha)=-c \alpha+\sigma^{2} \alpha^{2} / 2$. When $J(t)=i, i=1, \cdots, n$, $X^{f}(u, t)$ increases linearly at rate 1 , and thus with a Lévy exponent $\psi_{j}(\alpha)=\alpha$. Such a process is called Markov additive process - MAP (See Asmussen (2003) Chapter XI).

The process $J(t)$ describing the modulating state is a continuous time jump Markov process with a generator $\mathbf{Q}$, where

$$
\mathbf{Q}=\left(\begin{array}{cc}
-\lambda & \lambda \beta \\
\boldsymbol{t} & \boldsymbol{T}
\end{array}\right)
$$

Note that $\mathbf{Q}$ is an $n+1 \times n+1$ matrix, where $n$ is the number of phases in the phase time distribution. When the modulating state is $i, X^{f}(u, t)$ behaves as a Lévy process with exponent $\psi_{i}(\alpha)$. Let

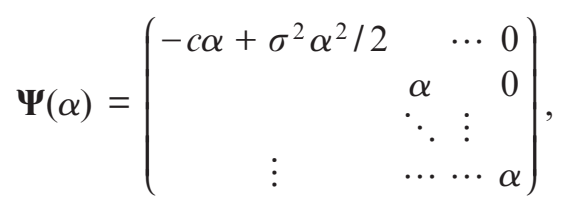

Let $\mathbf{K}$ be the following matrix:

$$
\mathbf{K}(\alpha)=\mathbf{Q}+\boldsymbol{\Psi}(\alpha) .
$$


Asmussen (2003), Chapter XI.2, shows that

$$
\mathbb{E}\left[e^{\alpha X^{f}(b, t)} ; J_{t}=j\right]=e^{t \mathbf{K}(\alpha)}, \text { for } \alpha \text { such that the expectation exists. }
$$

Let $\kappa(\alpha)$ be an eigenvalue of the matrix $\mathbf{K}(\alpha)$, and let $\mathbf{h}^{\alpha}$ be the corresponding right eigenvector. In our analysis we apply the multivariate version of Wald's martingale introduced in Lemma 2.1 of Asmussen and Kella (2000).

Lemma 2.5. Let $\alpha$ be a complex number such that $\mathbb{E}\left[e^{\alpha X^{f}(b, t)} ; J(t)=j \mid J(0)=i\right]$ exists, and let $\kappa(\alpha)$ and $\mathbf{h}^{\alpha}$ be eigenvalue and right eigenvector of the matrix $\mathbf{K}(\alpha)$, then with every initial distribution of $\left(X^{f}(b, 0), J(0)\right),\left\{e^{\alpha X^{f}(b, t)-t \kappa(\alpha)} h_{J(t)}^{\alpha}\right\}$ is a martingale.

\subsection{The probability $q(u)$}

In this section we apply the multidimensional Wald martingale to obtain the probability $q(u)$ - the probability that $X(u, t)$ hits the level 0 before the level $b$, note that $1-q(u)$ is the probability of ruin before any dividends are paid.

Let $q^{f}(u)$ be the probability that $X^{f}(u, t)$ reaches the level 0 before the level $b$. The construction of $X^{f}(u, t)$ implies that $q^{f}(u)=q(u)$. Let $\gamma(u, j)$ be the probability that the process $X^{f}(u, t)$, and hence $X(u, t)$, hits the level $b$ when the modulating state is $j, j=0, \cdots, n$, before reaching the level 0 . Thus, $\gamma(u, 0)$ is the probability of ruin before dividends are payed due to diffusion, and for $j=1$, $\cdots, n, \gamma(u, j)$ is the probability of ruin before dividends are paid due to a claim hitting the level 0 at phase $j$. Note that $q(u)=1-\sum_{j=0}^{n} \gamma(u, j)$.

Consider the equations $\operatorname{det}(\mathbf{K}(\alpha))=0$. Assume it has $n+2$ solutions $\alpha_{0}, \cdots$, $\alpha_{n+1}$, where $\alpha_{0}=0$, with eigenvector $\mathbf{h}^{0}=\mathbf{1}$. Let $\mathbf{h}^{i}$ be the eigenvectors corresponding to $\alpha_{i}$. Then by Lemma 2.5 , the process $e^{\alpha_{i} X^{f}(u, t)} h_{J(t)}^{j}$ is a martingale. Applying the optional sampling theorem to $T(u, 0, b)=\min (T(u, 0), T(u, b))$, we obtain:

$$
\sum_{j=0}^{n} e^{\alpha_{i} b} \gamma(u, j) h_{j}^{i}+q(u) h_{0}^{i}=e^{\alpha_{i}(b-u)} h_{0}^{i}
$$

Thus we obtain $n+2$ equations for $q(u)$, and $\gamma(u, 0) \cdots, \gamma(u, n)$.

\subsection{The expected dividends and the distribution of the deficit}

For $u \leq b$ and for $j=1, \cdots, n$, let $p(u, j)$ be the probability that the process $V(u, t)$ hits the level $b$ at phase $j$ and let $p(u, 0)$ be the probability that it hits the level $b$ by diffusion. Thus, $p(u, j)$ is the probability that $V^{f}(u, t)$ hits the level $b$ when the modulating state is $j, j=0, \cdots, n$. Note that $p(u, 0)$ is the probability that ruin 
occurs due to diffusion, while for $j=1, \cdots, n, p(u, j)$ is the probability that ruin occurs due to a claim that hits the level 0 at phase $j$.

Let $\mathbf{p}(u)=(p(u, 1), \cdots, p(u, n))$. Let $\zeta$ be the overflow of the process $V(u, t)$ at time $\tau(u)$, when it hits $b$. Thus $\zeta$ is the deficit at ruin. From the above discussion $\zeta$ is phase-type distributed with representation $((p(u, 0), \mathbf{p}(u)), \mathbf{T})$.

To find the probabilities $p(u, j)$, and the expected dividends $\boldsymbol{\ell}_{b}(u)$, we apply the Asmussen and Kella multidimensional martingale. Consider $V^{f}(u, t)$ as per (2.6). Asmussen and Kella (2000) proved that the process:

$$
\begin{aligned}
M(\alpha, t)= & \kappa(\alpha) \int_{0}^{t} e^{\alpha V^{f}(u, s)} d s h_{J(s)}^{\alpha} d s+e^{\alpha V^{f}(u, 0)} h_{J(0)}^{\alpha}-e^{\alpha V^{f}(u, t)} h_{J(t)}^{\alpha} \\
& +\alpha \int_{0}^{t} e^{\alpha V^{f}(u, t)} h_{J(s)}^{\alpha} d L^{f}(u, s)
\end{aligned}
$$

is a zero mean martingale. Note that $d L^{f}(u, s)>0$ implies that $J(s)=0$ and $V^{f}(u, s)=0$.

Substituting in (2.11) $\alpha=\alpha_{j}$, such that $\operatorname{det}\left(\mathbf{K}\left(\alpha_{j}\right)\right)=0, \kappa\left(\alpha_{j}\right)=0, J(0)=0$, and $V^{f}(u, 0)=b-u$, we obtain that the process

$$
e^{\alpha_{j}(b-u)} h_{0}^{j}-e^{\alpha_{j} V f(u, t)} h_{J(t)}^{j}+\alpha_{j} L^{f}(u, t) h_{0}
$$

is a zero mean martingale, $j=0, \cdots, n+1$. Applying the optional sampling theorem yields

$$
e^{\alpha_{j}(b-u)} h_{0}^{j}-e^{\alpha_{j} b} \sum_{k=0}^{n} h_{k}^{j} p(u, k)+\alpha_{j} \ell_{b}(u) h_{0}^{j}=0 .
$$

Note that the equation for $j=0$ is $\sum_{k=0}^{n} p(u, k)=1$. Thus we get $n+2$ equations for $p(u, 0), \cdots, p(u, n)$ and $\boldsymbol{\ell}_{b}(u)$.

\section{Conclusions}

a. When the initial reserve is $b$, the total dividends paid is exponentially distributed with mean $\boldsymbol{\ell}_{b}(b)$, where $\boldsymbol{\ell}_{b}(b)$ is found by solving (2.12) with $u=b$.

b. When the initial reserve is $u<b$ the total dividends paid is 0 with probability $1-q(u)$, and it is exponentially distributed with mean $\boldsymbol{\ell}_{b}(b)$ with probability $q(u)$, where $q(u)$ is obtained in Section 2.4.

The deficit at ruin. Let $\zeta$ be the deficit at ruin. $p(u, 0)$ is the probability that ruin occurs by diffusion, and $p(u, j)$ is the probability that ruin occurs when a claim crosses 0 at phase $j, j=1, \cdots, n$. Therefore the deficit at ruin is phase type with an atom $(p(u, 0), \mathbf{p}(u), \mathbf{T})$, where $\mathbf{p}(u)=(p(u, 1), \cdots, p(u, n))$. 


\subsection{The expected time to ruin}

Let $X(u, t)$ be the Lévy process as defined in the previous section, and let $V(u, t)$ be the reflected Lévy process. Note that $V\left(u, \tau_{b}(u)\right)=b+\zeta$, where $\zeta$ is the deficit at ruin. Applying Wald identity (see Asmussen (2003), p. 258-259),

$$
b-u+\mathbb{E}[X(1)] \mathbb{E}\left[\tau_{b}(u)\right]=b-u+(-c+\lambda \mathbb{E}[W]) \mathbb{E}\left[\tau_{b}(u)\right]=\mathbb{E}\left[X\left(u, \tau_{b}(u)\right)\right] .
$$

By substituting

$$
X\left(u, \tau_{b}(u)\right)=V\left(u, \tau_{b}(u)\right)-L\left(u, \tau_{b}(u)\right)=b+\zeta-L\left(u, \tau_{b}(u)\right),
$$

taking expectations, and applying (2.5), we obtain that

$$
\mathbb{E}\left[\tau_{b}(u)\right]=\frac{\ell_{b}(u)-\mathbb{E}[\zeta]-u}{c-\lambda \mathbb{E}[W]}=\frac{\ell_{b}(u)+\mathbf{p}(u) \mathbf{T}^{-1} \mathbf{1}-u}{c+\lambda \boldsymbol{\beta} \mathbf{T}^{-1} \mathbf{1}} .
$$

Thus we can obtain the expected time to ruin.

\subsection{The algorithm}

We summarize the procedure for one dividend barrier. Assume that the claim size distribution is phase type with representation $(\boldsymbol{\beta}, \mathbf{T})$. Then:

(i) Construct the matrix $\mathbf{K}(\alpha)$, as per (2.9).

(ii) Solve the equations $\operatorname{det}(\mathbf{K}(\alpha))=0$ to obtain the $n+2$ solutions $\alpha_{0}, \cdots, \alpha_{n+1}$.

(iii) For each $\alpha_{j}$ find the non-null vector $\mathbf{h}^{j}$, such that $\mathbf{K}\left(\alpha_{j}\right) \mathbf{h}^{j}=\mathbf{0}$.

(iv) Solve the set of $n+2$ equations in (2.10), find $q(u)$-probability of reaching $b$ before ruin, and the probabilities $\gamma(u, j)$ of ruin in phase $j$ before dividends payment.

(v) Solve the set of $n+2$ equations in (2.12) to find the expected dividends and the probabilities $p(u, 0)$, and $p(u, j), j=1, \cdots, n$ of ruin due to diffusion or due to a claim at phase $j$, respectively.

(vi) The deficit at ruin is phase type with an atom $p(u, 0)]$ at 0 , and with representation $((p(u, 0), \mathbf{p}(u)), \mathbf{T})$.

(vii) The expected time to ruin is as per (2.13).

\subsection{Detailed example}

In this example we imply the last procedure step by step on the following data: $u=20, b=50, c=0.7, \lambda=1$. We chose the claim amount distribution to be 
a phase type distribution that approximates the distribution of $\min \left(e^{v W-v^{2} / 2}, 2\right)$, where $W$ is a standard normal random variable, i.e. our phase type distribution approximates a truncated lognormal distribution. This distribution fits the distribution of claim amount of some Swedish fire insurance data. The approximation is presented in Asmussen and Rolski (1991). When $v=1.8$ the parameters of the fitted phase type distribution are:

$$
\boldsymbol{\beta}=(0.5614,0.4386), \quad \mathbf{T}=\left(\begin{array}{rr}
-8.640 & 1.997 \\
0.101 & -1.095
\end{array}\right), \quad \mathbf{t}=\left(\begin{array}{l}
6.643 \\
0.994
\end{array}\right)
$$

The expected claim amount is $\mathbb{E}(W)=0.6015$.

(i)

$$
\begin{aligned}
& \mathbf{Q}=\left(\begin{array}{rrr}
-1 & 0.5614 & 0.4386 \\
6.643 & -8.640 & 1.997 \\
0.994 & 0.101 & -1.095
\end{array}\right) \\
& \boldsymbol{\Psi}(\alpha)=\left(\begin{array}{ccc}
-0.7 \alpha+\alpha^{2} / 2 & 0 & 0 \\
0 & \alpha & 0 \\
0 & 0 & \alpha
\end{array}\right) \\
& \mathbf{K}(\alpha)=\left(\begin{array}{ccc}
-0.7 \alpha+1 / 2 \alpha^{2}-1 & 0.5614 & 0.4386 \\
6.643 & \alpha-8.640 & 1.997 \\
0.994 & 0.101 & \alpha-1.095
\end{array}\right)
\end{aligned}
$$

(ii) Solve $\operatorname{det}(\mathbf{K})(\alpha)=\alpha\left(0.5 \alpha^{3}-5.5675 \alpha^{2}+10.4440 \alpha-0.9117\right)=0$. Solutions: $\alpha_{0}=0, \alpha_{1}=0.0917, \alpha_{2}=2.2638, \alpha_{3}=8.7795$

(iii) Solve $\mathbf{K}\left(\alpha_{j}\right) \mathbf{h}^{j}=0$ yields

$$
\mathbf{h}^{0}=\left(\begin{array}{l}
1 \\
1 \\
1
\end{array}\right), \quad \mathbf{h}^{1}=\left(\begin{array}{l}
0.5534 \\
0.5716 \\
0.6058
\end{array}\right), \quad \mathbf{h}^{2}=\left(\begin{array}{c}
-0.6443 \\
-0.4865 \\
0.5900
\end{array}\right), \quad \mathbf{h}^{3}=\left(\begin{array}{c}
0.0177 \\
-0.9998 \\
0.0108
\end{array}\right)
$$

(iv) Solve the set of equations (2.10) to find $q(u)$, and $\gamma(20, j), j=0,1,2$. The solution is: $\gamma(20,0)=0.0670, \gamma(20)=0.0020,, \gamma(20,2)=0.0075$, and $q(20)=0.8562$.

Note that $q(20)$ is the probability of reaching $b=50$ before ruin, and $\gamma(20, j), j=1,2$ is the probability of ruin before reaching $b$ due to a claim in phase $j$, and $\gamma(20,0)$ is the probability of ruin before reaching $b$, due to diffusion. 
(v) Solve the set of equations (2.12). The solution yields that the probability of ruin due to diffusion is: $p(20,0)=0.4659$, the probability of ruin due to a claim in phase 1 is $p(20,1)=0.0139$, and due to a claim at phase 2 is $p(20,2)=0.5202$. The expected dividends $\ell_{50}(20)=953.0$. Thus,

(vi) The deficit at ruin is phase type with atom $p(20,0)=0.4659$ at 0 , and representation $(0.0139,0.5202), \mathbf{T})$.

(vii) Substituting $\ell_{50}(20), p(20,1), p(20,2)$ in (2.13) yields that the expected time to ruin is $\mathbb{E}\left(\tau_{b}(u)\right)=9470.3$.

\subsection{Numerical example}

Example I. In this section the data and the claim amount distribution is as in 2.8. In tables 1 and 2 we show the expected dividends paid and the expected time to ruin for different barrier levels and three value of volatility $\sigma$.

TABLE 1

THE EXPECTED DIVIDENDS

\begin{tabular}{l|l|l|l}
\hline \hline$b$ & $\sigma=0.5$ & $\sigma=1$ & $\sigma=1.5$ \\
\hline 20 & 117 & 61 & 39 \\
30 & 456 & 152 & 70 \\
40 & 1773 & 381 & 126 \\
50 & 6894 & 953 & 227 \\
60 & 26806 & 2385 & 408 \\
70 & 104229 & 5970 & 733 \\
80 & 405269 & 14943 & 1317 \\
\hline \hline
\end{tabular}

TABLE 2

THE EXPECTED TIME TO RUIN

\begin{tabular}{l|l|l|l}
\hline \hline$b$ & $\sigma=0.5$ & $\sigma=1$ & $\sigma=1.5$ \\
\hline 20 & 980 & 409 & 191 \\
30 & 4420 & 1337 & 507 \\
40 & 17796 & 3659 & 1076 \\
50 & 69803 & 9470 & 2098 \\
60 & 272021 & 24016 & 3935 \\
70 & 1058298 & 60423 & 7237 \\
80 & 4115548 & 151548 & 13171 \\
\hline \hline
\end{tabular}

Example II. In the next example we apply the algorithm to the case where the volatility coefficient in the log-normal distribution is $v=0.8$. In this case the parameters of the phase type distribution that approximates the lognormal are as follows (Asmussen and Rolski (1991)):

$\boldsymbol{\beta}=(0.9731,0.0152,0.0106,0.001), \mathbf{T}=\left(\begin{array}{rrrr}-28.648 & 28.532 & 0.089 & 0.027 \\ 0.102 & -8.255 & 8.063 & 0.086 \\ 0.113 & 0.107 & -5.807 & 5.296 \\ 0.100 & 0.102 & 0.111 & -2.176\end{array}\right)$

In this case $\mathbb{E}(W)=0.8807$. We assume that $\lambda=1$, and $c=1$. The results for this data are exhibited in Tables 3 and 4. 
TABLE 3

THE EXPECTED DIVIDENDS

\begin{tabular}{l|l|l|l}
\hline \hline$b$ & $\sigma=0.5$ & $\sigma=1$ & $\sigma=1.5$ \\
\hline 20 & 146 & 72 & 44 \\
30 & 679 & 206 & 87 \\
40 & 3150 & 591 & 558 \\
50 & 14621 & 1695 & 342 \\
60 & 67849 & 4864 & 678 \\
70 & 314401 & 13961 & 1344 \\
80 & 1447596 & 40063 & 22189 \\
\hline \hline
\end{tabular}

TABLE 4

THE EXPECTED TIME TO RUIN

\begin{tabular}{l|l|l|l}
\hline \hline$b$ & $\sigma=0.5$ & $\sigma=1$ & $\sigma=1.5$ \\
\hline 20 & 1054 & 430 & 197 \\
30 & 5518 & 1555 & 558 \\
40 & 26240 & 1781 & 1274 \\
50 & 122424 & 14041 & 2694 \\
60 & 568702 & 40617 & 5512 \\
70 & 2635889 & 118887 & 11102 \\
80 & 12137038 & 335735 & 22190 \\
\hline \hline
\end{tabular}

In both examples we see that the expected dividends and the expected time to ruin are very sensitive to the the volatility coefficient of the Brownian motion. Both decrease as the volatility coefficient increases.

\section{Model II}

\subsection{Model description}

Let $a, b$ be numbers, such that $b>a>u$. Whenever the risk process reaches the level $b$, dividends are paid at a rate $d<c$. Let $c_{1}=c-d$. The dividends are paid until the process reaches the level $a$. Thus, if the surplus reaches the level $b$ before ruin, dividends are paid, until the surplus down-crosses the level $a$, and so on. We consider two cases: 1. $c_{1}<\lambda \mathbb{E}[W]$, and 2. $c_{1}>\lambda \mathbb{E}[W]$. In the first case ruin occurs with probability 1 , and thus we will obtain the expected dividends paid until ruin occurs, and the expected time to ruin. In the second case we will find the ruin probability. Unless assuming otherwise, we consider the first case.

\subsection{The expected dividends}

Consider the risk process described above, with initial reserve $u$. Let $e_{1}<o_{1}<$ $e_{2}<o_{2}<\cdots . e_{j}$ is the $j$ th time that the process crosses the level $b$ from below and $o_{j}$ is the first time after $e_{j}$ that the process crosses the level $a$ from above. Thus $e_{j}-o_{j}$ is the duration of the $j$-th period of dividend payments. Assume that the surplus process $R(u, t)$ reaches the level $b$ before ruin. From that point until the reserve reaches level $a$, it behaves as a risk process $R_{1,1}(b-a, t)=b-$ $a+c_{1} t-\sum_{j=1}^{N(t)} W_{j}$. Let $X_{1}(t)=b-a-R_{1}(b-a, t)$. Let $\tau_{1, j}(b-a)=o_{j}-e_{j}$. Thus $\tau_{1, j}(b-a)$ is the time to ruin for the process $R_{1, j}(b-a, t)$, with $R_{1, j}(b-a, t)$ is the risk process between $e_{j}$ and $o_{j}$. Since $c_{1}<\lambda \mathbb{E}[W]$, the expected time to ruin is finite. Let $\zeta_{1, j}$ be the deficit at ruin for the process $R_{1, j}(b-a, t)$. Note that 
$R_{1, j}, \zeta_{1, j}$ and $\tau_{1, j} j=1,2, \cdots$ are probabilistically identical to $R_{1,1}, \zeta_{1,1}$ and $\tau_{1,1}$, respectively. Thus we will use the notation $R_{1}, \zeta_{1}$ and $\tau_{1}$, respectively. Wald equality implies that:

$$
\mathbb{E}\left[\tau_{1}(b-a)\right]=\frac{(b-a)+\mathbb{E}\left[\zeta_{1}\right]}{\lambda \mathbb{E}[W]-c_{1}}
$$

The deficit at ruin $\zeta_{1}$ for the process $R_{1}$ is phase-type distributed with an atom $\eta_{0}$ at zero, and with representation $\left(\left(\eta_{0}, \boldsymbol{\eta}\right), \mathbf{T}\right)$, where $\boldsymbol{\eta}=\left(\eta_{1}, \cdots, \eta_{n}\right)$. To find $\left(\eta_{0}, \boldsymbol{\eta}\right)$, we consider the fluid version, $X_{1}^{f}(t)$, of $X_{1}(t)$. Thus, $\eta_{j}$ is the probability that $X_{1}(t)$ hits the level $b-a$ at state $j, j=0, \cdots, n, \eta_{0}$ is the probability in the process $R_{1}(b-a, t)$ ruin occurs due to diffusion, while for $j=1, \cdots, n, \eta_{j}$ is the probability of ruin due to a claim in state $j . X_{1}^{f}(t)$ is a MAP, as described in Section 2.3. The modulating states are $0, \cdots, n$. When in state 0 the process behaves as Brownian motion with a drift $-c_{1}$, and variance $\sigma^{2}$, and when in state $j=1, \cdots, n$, the process increases linearly at rate 1 . Let $J(t)$ be a jump Markov process describing the modulating state; the generator of $J(t)$ is $\mathbf{Q}$, as per (2.7).

$\mathbb{E}\left[e^{\alpha X_{1}^{f}(t)} ; J(t)=j \mid J(0)=i\right]=e^{t \mathbf{K}_{1}}(\alpha)$, where $\mathbf{K}_{1}(\alpha)=\mathbf{Q}+\boldsymbol{\Psi}_{1}(\alpha)$, and $\boldsymbol{\Psi}_{1}(\alpha)$ is as $\Psi(\alpha)$ in (2.8), where $c$ is replaced by $c_{1}$. Assume that the equation $\operatorname{det}\left(\mathbf{K}_{1}(\alpha)\right)=0$ has $n+2$ different roots $\alpha_{1,0}, \cdots, \alpha_{1, n+1}$. Note that one of the roots, let's say $\alpha_{1,0}$ is 0 , with corresponding eigenvector equal to $\mathbf{1}$. Let $\mathbf{h}_{1}^{j}$ be the eigenvectors of $\mathbf{K}\left(\alpha_{1, j}\right)$, that is non-zero vectors such that

$$
\mathbf{K}\left(\alpha_{1, j}\right) \mathbf{h}_{1}^{j}=\mathbf{0} .
$$

The assumption that $c_{1}<\lambda \mathbb{E}[W]$ implies that $\mathbb{E}\left[\tau_{1}(b-a)\right]<\infty$. As in Section 2.4, we apply the multidimensional Wald martingale in Lemma 2.5 , and the optional sampling theorem, to obtain the following equations for $\left(\eta_{0}, \boldsymbol{\eta}\right)$ :

$$
\sum_{j=0}^{n} e^{\alpha_{1, i}(b-a)} \eta_{j} h_{1, j}^{i}=h_{1,0}^{i} \quad i=0, \cdots, n+1 .
$$

Note that for $i=0$, equation (3.2) reads: $\sum_{j=0}^{n} \eta_{j}=1$.

Corollary 3.1. The deficit at ruin, $\zeta_{1}$, of the risk process $R_{1}(b-a, t)$ is phase-type with representation $\left(\left(\eta_{0}, \boldsymbol{\eta}\right), \mathbf{T}\right)$, where $\left(\eta_{0}, \boldsymbol{\eta}\right)$ is as per (3.2). Thus

$$
\mathbb{E}\left[\zeta_{1}\right]=-\boldsymbol{\eta} \mathbf{T}^{-1} \mathbf{1} .
$$

The risk process $R(u, t)$ starts at level $u$. With probability $q(u)$ it reaches the level $b$ before the level 0 , and with probability $1-q(u)$ ruin occurs before the process reaches $b$ and no dividends are paid. In the case that level $b$ is reached before ruin, the risk process behaves as $R_{1}(b-a)$ during the period $\tau_{1}=e_{1}-o_{1}$. 
From time $e_{1}$ to $o_{2}$, the risk process behaves as $R_{2,1}(t)$. Similarly, for $j=1,2, \ldots$, from $e_{j}$ to $o_{j+1}$ the surplus process $R_{2, j}(t)$ is below $b$, and behaves as $R_{2}(t)$, where

$$
R_{2}(t)=a-\zeta_{1}+c t+\sigma B(t)-\sum_{k=1}^{N(t)} W_{k}
$$

Let $X_{2}(t)=b-R_{2}(t)$. The process $X_{2}$ is a Lévy process which is statistically identical to the process $X\left(a-\zeta_{1}, t\right)$. Let $\rho$ be the probability that $X_{2}(t)$ reaches the level 0 before the level $b$. Clearly this probability is 0 , if $\zeta_{1}>a$ (i.e. just before ruin the surplus process is above $a$, dividends are paid, and ruin occurs due to a claim). Let $A$ be the event $A=\left(\zeta_{1}<a\right)$. Let $\xi$ be a random variable with distribution function $F_{\xi}$, where

$$
F_{\xi}(x)=P\left(\zeta_{1} \leq x \mid \zeta_{1}<a\right) .
$$

Let $\tilde{T}(0)$ and $\tilde{T}(b)$ be the first time that the process $X_{2}(t)$ hits 0 and $b$, respectively, given $A$. Let $\hat{\rho}$ be the probability that the process $X_{2}(t)$ hits 0 before $b$, given $A$. Note that if $\tilde{T}(b)<\tilde{T}(0)$, then ruin occurs. Note further that $X_{2}(t)$ hits 0 due to a diffusion while it may hit $b$ due to a diffusion (state 0 ), or at phase $k, k=1, \cdots, n$.

Let $\tilde{\omega}(0)$ be the probability that $X_{2}(t)$ hits level $b$ before 0 due to diffusion, and let $\tilde{\omega}(j)$ be the probability that it hits level $b$ before level 0 at phase $j$, $j=1, \cdots, n$, given $A$. To obtain $\hat{\rho}$ and $\tilde{\omega}(j) j=0, \cdots, n$, we consider the fluid version $X_{2}^{f}(t)$ of $X_{2}(t)$ and apply the multidimensional Wald martingale as per Lemma 2.5

$$
\sum_{j=0}^{n} e^{\alpha_{1} b} \tilde{\omega}(j) h_{j}^{i}+\hat{\rho} h_{0}^{i}=\mathbb{E} e^{\alpha_{i}(b-a+\xi)} h_{0}^{i},
$$

where $\left(\alpha_{i}, i=0, \cdots, n+1\right)$ and $\left(h_{j}^{i}, i=0, \cdots, n+1, j=0, \cdots, n\right)$ are as in (2.10), and

$$
\begin{aligned}
\mathbb{E} e^{\alpha_{i}(b-a+\xi)} & =e^{\alpha_{i}(b-a)} \frac{\eta(0)+\int_{0}^{a} e^{\alpha_{i} s} \boldsymbol{\eta} e^{\mathbf{T} s} \mathbf{t} d s}{1-\boldsymbol{\eta} e^{\mathbf{T} a} \mathbf{1}} \\
& =e^{\alpha_{i}(b-a)} \frac{\eta(0)+\boldsymbol{\eta}\left(\mathbf{T}+\alpha_{i} \mathbf{I}\right)^{-1}\left(e^{\left(\mathbf{T}+\alpha_{i} \mathbf{I}\right) a}-\mathbf{I}\right) \mathbf{t}}{1-\boldsymbol{\eta} e^{\mathbf{T} a} \mathbf{1}} .
\end{aligned}
$$

Let $\omega_{0}$ be the probability that $X_{2}(t)$ hits level $b$ before 0 due to diffusion, let $\omega(k)$ be the probability it hits the level $b$ before level 0 at phase $k, k=1, \cdots, n$, and let $\rho$ be the probability that $X_{2}(t)$ hits 0 before $b$ (by diffusion). Then 


$$
\begin{aligned}
\omega_{0} & =P\left(\zeta_{1} \leq a\right) \tilde{\omega}_{0} \\
\omega_{k} & =P\left(\zeta_{1} \leq a\right) \tilde{\omega}_{k}+\boldsymbol{\eta} e \mathbf{T}^{a} \mathbf{1}_{k}, k=1, \cdots, n \\
\rho & =P\left(\zeta_{1} \leq a\right) \hat{\rho}
\end{aligned}
$$

where $\mathbf{1}_{k}$ is an $n$ dimensional column vector with all components 0 except the $j$ th which is 1 . Let $\omega=\left(\omega_{1}, \cdots, \omega_{n}\right)$. Thus $\omega_{0}$ is the probability of ruin (for the process $R_{2}$ ) due to diffusion, and $\omega_{k} k=1, \cdots, n$ is the probability of ruin due to a claim at phase $k . \rho$ is the probability that $R_{2}$ reaches level $b$ before ruin.

\subsubsection{The deficit at ruin}

Assume that the initial reserve is $u$. With probability $\gamma(u, 0)$, ruin occurs before any dividends' payments due to a diffusion, and with probability $\gamma(u, j), j=1, \cdots, n$ ruin occurs before any dividends' payments due to a claim at phase $j \cdot \gamma(u, 0)$, and $\gamma(u)=(\gamma(u, 1), \cdots, \gamma(u, n))$ are as per (2.10). In this case the deficit at ruin is phase type with representation $((\gamma(u, 0), \gamma(u)), \mathbf{T})$. With probability $q(u)$ the deficit at ruin is phase type with representation $\left(\omega_{0} /(1-\rho), \omega /(1-\rho)\right)$, where $\omega_{0}, \omega=\left(\omega_{1}, \cdots, \omega_{n}\right)$ and $\rho$ are as per (3.8). Thus the deficit at ruin is phase-type with representation

$$
\left(\left(\gamma(u, 0)+q(u) \frac{\omega_{0}}{1-\rho}, \gamma(u)+\frac{q(u)}{1-\rho} \omega\right), \mathbf{T}\right) .
$$

\subsubsection{The amount of dividends}

Define the $j$-th cycle, $C(j)$, as the time elapses from $e_{j}$ to $e_{j+1}$. Clearly the cycles are i.i.d distributed as $C$. Let $C(u, j)$ be the time that elapses from $e_{j}$ to $o_{j}$, and $C(d, j)$ be the time that elapses from $o_{j}$ to $e_{j+1}$. Note that $C(u, j)$ is statistically identical to $\tau_{1}(b-a)$, and thus $\mathbb{E} C(u, j)$ is given by substituting (3.3) in (3.1). The expected dividend paid in one cycle is $d \mathbb{E}\left[\tau_{1}(b-a)\right]$. Let $\mathcal{N}$ be the number of cycles until ruin occurs. Then,

$$
\begin{aligned}
& P(\mathcal{N}=0)=1-q(u) \\
& P(\mathcal{N}=j)=q(u) \rho^{j-1}(1-\rho) j=1,2, \cdots .
\end{aligned}
$$

Thus

$$
\mathbb{E}[\mathcal{N}]=\frac{q(u)}{1-\rho} .
$$

Corollary 3.2. The expected dividends paid until ruin occurs are:

$$
d \mathbb{E}[\mathcal{N}] \mathbb{E}[C(u, 1)]=\frac{d q(u)}{1-\rho} \frac{b-a-\boldsymbol{\eta} \mathbf{T}^{-1} \mathbf{1}}{\lambda \mathbb{E}[W]-c_{1}} .
$$




\subsubsection{The expected time to ruin}

Let $\tau(u)$ be the time to ruin. Then

$\tau(u)=\min (T(u, 0), T(u, b))+I(T(u, 0)<T(u, b))\left(\sum_{j=1}^{\mathcal{K}}(C(u, j)+C(d, j))-\Delta\right)$,

where $I$ is the indicator function, and $\Delta$ is the time that elapses from the moment that ruin occurs in the $\mathcal{N}$ th cycle until the end of this cycle, i.e., until $e_{\mathfrak{x}+1}$.

If the event $(T(u, 0)<T(u, b))$ occurs, then $X(u, T(u, 0, b))=0$. The process $X(u, t)$ reaches the level 0 before the level $b$. Recall that $P(T(u, 0)<T(u, b))=$ $q(u)$.

If $(T(u, 0)>T(u, b))$, then $X(u, t)$ hits level $b$ before level 0 , and ruin occurs before any dividends are paid. In this case $X(u, T(u, 0, b))=b+\zeta$, where $\zeta$ is the deficit at ruin. Given that $(T(u, 0)>T(u, b))$, the deficit at ruin- $\zeta$ is phase type with representation, $(\gamma(u, 0) /(1-q(u)), \gamma /(1-q(u))$, T), where $\gamma(u, 0)$ and $\gamma(u)=$ $(\gamma(u, 1), \cdots, \gamma(u, n))$ are given in (2.10). Wald identity implies that

$$
\mathbb{E}[T(u, 0, b)]=\frac{q(u) b-u+\gamma(u) \mathbf{T}^{-1} \mathbf{1}}{c-\lambda \mathbb{E}[W]} .
$$

The random variable $I(T(0, u)<T(b, u))$ is independent of the evolution of the process after time $T(u, 0, b)$ and $P(I(T(0)<T(b))=1)=q(u)$. By Corollary 3.1

$$
\mathbb{E}[C(u, j)]=\frac{(b-a)+\mathbb{E}\left[\zeta_{1}\right]}{\lambda \mathbb{E}[W]-c_{1}}=\frac{(b-a)-\boldsymbol{\eta} \mathbf{T}^{-1} \mathbf{1}}{-\lambda \boldsymbol{\beta} \mathbf{T}^{-1} \mathbf{1}-c_{1}},
$$

where $\boldsymbol{\eta}$ is as per (3.2). $C(d, j)$ is the time that elapses from $o_{j}$ where $X_{2}(0)=b$ $-a+\zeta_{1}$ until $e_{j+1}$. Applying the Wald identity, we obtain

$$
\mathbb{E}[C(d, j)]=\frac{b-a+\mathbb{E}\left[\zeta_{1}\right]}{c-\lambda \mathbb{E}[W]} .
$$

$\Delta$ is the time from the moment that $X_{2}(t)$ up-crosses level $b$ until it hits 0 in the $\mathcal{N}$ cycle. The overflow of the process $X_{\mathcal{X}}(t)$ above $b$ is just the deficit at ruin given that ruin occurs. Let $\zeta$ be a random variable distributed as the deficit at ruin given that ruin occurs in the $\mathcal{N}$ cycle. Then $\zeta$ is phase-type distributed with representation $\left(\omega_{0} /(1-\rho), \omega /(1-\rho), \mathbf{T}\right)$. Applying the Wald identity we obtain that:

$$
\mathbf{E}[\Delta]=\frac{b-(\omega /(1-\rho)) \mathbf{T}^{-1} \mathbf{1}}{c-\lambda \mathbb{E}[W]}
$$


Corollary 3.3. The expected time to ruin $\mathbb{E}[\tau(u)]$ is as follows:

$$
\mathbb{E}[\tau(u)]=\mathbb{E}[T(u, 0, b)]+q(u)(\mathbb{E}[\mathcal{N}](\mathbb{E}[C(u, 1)]+\mathbb{E}[C(d, 1)])-\mathbb{E}[\Delta])
$$

\subsubsection{Detailed example}

In this example we implement the last procedure step by step to the following data: $c=0.7, \lambda=1, c_{1}=0.5$, (i.e. dividends are paid at rate 0.2 ), $u=20, b=50$, $a=40$, and the claim distribution is two dimensional phase type as in section 2.8. In the following procedure we add only the additional steps to those in section 2.8 .

1.

$$
\begin{gathered}
\boldsymbol{\Psi}_{1}(\alpha)=\left(\begin{array}{ccc}
-c_{1} \alpha+\sigma^{2} \alpha^{2} / 2 & 0 & 0 \\
0 & \alpha & 0 \\
0 & 0 & \alpha
\end{array}\right)=\left(\begin{array}{ccc}
-0.7 \alpha+\alpha^{2} / 2 & 0 & 0 \\
0 & \alpha & 0 \\
0 & 0 & \alpha
\end{array}\right) \\
\mathbf{K}_{1}(\alpha)=\left(\begin{array}{ccc}
-0.5 \alpha+1 / 2 \alpha^{2}-1 & 0.5614 & 0.4386 \\
6.643 & \alpha-8.64 & 1.997 \\
0.994 & 0.101 & \alpha-1.095
\end{array}\right) .
\end{gathered}
$$

2. Solve $\operatorname{det}\left(\mathbf{K}_{1}(\alpha)\right)=\alpha\left(0.5 \alpha^{3}-5.3675 \alpha^{2}+8.49705 \alpha-0.94010\right)=0$. Solutions: $\alpha_{1,0}=0, \alpha_{1,1}=-0.104, \alpha_{1,2}=2.065, \alpha_{1,3}=8.774$.

3. For $j=0,1,2,3$, solve $\mathbf{K}_{1}\left(\alpha_{j}\right) \mathbf{h}_{1}^{j}=0$. The solutions are:

$$
\mathbf{h}_{1}^{0}=\left(\begin{array}{l}
1 \\
1 \\
1
\end{array}\right), \mathbf{h}_{1}^{1}=\left(\begin{array}{l}
0.60128 \\
0.58189 \\
0.54760
\end{array}\right), \mathbf{h}_{1}^{2}=\left(\begin{array}{c}
-0.61329 \\
-0.41565,0.67164
\end{array}\right), \mathbf{h}_{1}^{3}=\left(\begin{array}{c}
0.01681 \\
-0.998 \\
0.01097
\end{array}\right)
$$

4. Solve the set of equations (3.2) to find $\eta_{0}$ - the probability that ruin in the risk process $R_{1}(b-a)$ occurs due to diffusion, and $\eta_{j}$ - the probability that ruin in the risk process $R_{1}(b-a)$ occurs due to claim in phase $j, j=1,2$. The solution is: $\eta_{0}=0.51102, \eta_{1}=0.01381$, and $\eta_{2}=0.47517$. Thus the deficit at ruin, $\zeta_{1}$, of the surplus process $R_{1}$, is phase type with representation $((0.0138,0.47517), \mathbf{T})$.

5. Let $\xi$ be a random variable distributed as the conditional distribution of $\zeta_{1}$ given $\zeta_{1} \leq a$. To find the right hand side of 3.6 we have to substitute the solutions $\alpha_{i}, i=0,1,2,3$ as per section 2.8 .

6. Solve the set of equations (3.6) to obtain $\tilde{\omega}_{i}, i=0,1,2$ - the probability that ruin occurs due to diffusion, or due to to a claim hitting 0 at phase $i, i=$ 1,2 , and $\hat{\rho}$, the probability that the process reaches the level $b$ before ruin. All this probabilities are conditional probabilities given that $R_{2}$ starts with 
reserve $a-\zeta_{1}>0$. The solution is: $\tilde{\omega}_{0}=0.00738, \tilde{\omega}_{1}=0.00022, \tilde{\omega}_{1}=0.00824$, $\hat{\rho}=0.98417$.

7. Find $\omega_{i}, i=0,1,2$ - the ruin probability for $R_{2}$ due to diffusion or due to a claim in phase $i$. In this example $\operatorname{Pr}\left(\zeta_{1} \leq a\right) \approx 1$, thus $\omega_{i} \approx \tilde{\omega}_{i}, i=0,1,2$, and $\rho \approx \hat{\rho}$.

8. The deficit at ruin is phase type with parameter 0 with probability $\left(\gamma_{0}+\right.$ $\left.q(u) \omega_{0} /(1-\rho), \gamma(u)+q(u) /(1-\rho) \omega, \mathbf{T}\right)$, with $\gamma_{0}+q(u) \omega_{0} /(1-\rho)=0.46615$, $\gamma_{1}+q(u) \omega_{1} /(1-\rho)=0.0139$, and with probability $\gamma_{2}+q(u) \omega_{2} /(1-\rho)=$ 0.520489 .

9. The expected number of cycles is as per (3.9), $\mathbb{E}[\mathcal{N}]=54.13$.

10. The expected time dividends are paid per cycle is as per $(3.1), \mathbb{E}[C(u, 1)]=$ 103.

11. The expected dividends $d \mathbb{E}[\mathcal{N}] \mathbb{E}[C(u, 1)]=1113.56$.

12. The expected time until the risk process hits $b$ or 0 is: $\mathbb{E}[T(u, 0, b)]=231$.

13. The expected time in one cycle that the process is below $b$ is $\mathbb{E}[C(d, 1)]=$ 106.15.

14. The expected time from the moment of ruin until the surplus reaches level $b$ is $\mathbb{E}[\Delta]=513$.

15. The expected time to ruin:

$$
\mathbb{E}[T(u, 0, b)]+q(u)(\mathbb{E}[\mathcal{N}](\mathbb{E}[C(u, 1)]+\mathbb{E}[C(d, 1))-\mathbb{E}[\Delta]=11101 .
$$

\subsection{Numerical example}

Example III. We imply the algorithm to data in 3.2.4 for different values of $b, a=0.8 b$, and $\sigma$.

TABLE 5

THE EXPECTED DIVIDENDS

\begin{tabular}{l|l|l|l}
\hline \hline$b$ & $\sigma=0.5$ & $\sigma=1$ & $\sigma=1.5$ \\
\hline 20 & 162 & 96 & 67 \\
30 & 542 & 218 & 114 \\
40 & 1801 & 494 & 192 \\
50 & 5945 & 1134 & 325 \\
60 & 19509 & 2504 & 548 \\
70 & 63664 & 5616 & 921 \\
80 & 206671 & 12560 & 1548 \\
\hline \hline
\end{tabular}

TABLE 6

THE EXPECTED TIME TO RUIN

\begin{tabular}{l|l|l|l}
\hline \hline$b$ & $\sigma=0.5$ & $\sigma=1$ & $\sigma=1.5$ \\
\hline 20 & 1433 & 768 & 479 \\
30 & 5294 & 2008 & 953 \\
40 & 18978 & 4806 & 1751 \\
50 & 60165 & 11101 & 3095 \\
60 & 197915 & 25223 & 5335 \\
70 & 646337 & 56822 & 9150 \\
80 & 2098661 & 127345 & 15517 \\
\hline \hline
\end{tabular}


Example IV. In this example we imply the algorithm to the following data: $c=1, \lambda=1, u=20, a=0.8 b$, and claim size distribution is as in Example II in section 2.9.

TABLE 7

THE EXPECTED DIVIDENDS

\begin{tabular}{l|l|l|l}
\hline \hline$b$ & $\sigma=0.5$ & $\sigma=1$ & $\sigma=1.5$ \\
\hline 20 & 174 & 100 & 68 \\
30 & 649 & 249 & 123 \\
40 & 2398 & 611 & 222 \\
50 & 8799 & 1503 & 399 \\
60 & 32066 & 3683 & 717 \\
70 & 116118 & 8995 & 1288 \\
80 & 417988 & 21897 & 2310 \\
\hline \hline
\end{tabular}

TABLE 8

THE EXPECTED TIME TO RUIN

\begin{tabular}{l|l|l|l}
\hline \hline$b$ & $\sigma=0.5$ & $\sigma=1$ & $\sigma=1.5$ \\
\hline 20 & 1336 & 692 & 416 \\
30 & 5461 & 1977 & 893 \\
40 & 20658 & 5136 & 1752 \\
50 & 76263 & 12883 & 3294 \\
60 & 278390 & 31821 & 6062 \\
70 & 1008576 & 77966 & 11021 \\
80 & 3631033 & 190053 & 19895 \\
\hline \hline
\end{tabular}

Examples III and IV exhibit the same phenomena as Examples I and II of model 1 . The amount of dividends and the time to ruin are very sensitive to the volatility coefficient of the Brown motion. Both decrease as $\sigma$ increase.

\subsection{The ruin probability when $c_{1}>\lambda \mathbb{E}[W]$}

Assume that $c_{1}>\lambda \mathbb{E}[W]$. In this case the ruin probability is less than 1 . Let $\varphi_{1}(b-a)$ be the ruin probability for the risk process $R_{1}$, and let $\zeta_{1}$ be distributed as the deficit at ruin, given that ruin occurs.

The ruin probability $\varphi_{b}(u)$ can be written as follows:

$$
\begin{aligned}
\varphi_{b}(u) & =1-q(u)+q(u) \sum_{j=1}^{\infty}\left(\varphi_{1}(b-a) \rho\right)^{j-1} \varphi_{1}(b-a)(1-\rho) \\
& =1-q(u)+q(u) \frac{\varphi_{1}(b-a)(1-\rho)}{1-\varphi_{1}(b-a) \rho} .
\end{aligned}
$$

Recall that $1-q(u)$ is the probability that ruin occurs before reaching the dividend barrier. With probability $q(u)$ the dividend barrier is reached before ruin occurs. $1-\rho$ is the probability that ruin occurs, given that ruin occurred at the process $R_{1}$. Thus, $\varphi_{1}(b-a) \rho$ is the probability of reaching the dividend barrier before ruin occurs, after ruin occurs in $R_{1}$.

To obtain $\varphi_{1}(b-a)$, and the distribution of $\zeta_{1}$, we apply the result obtained by Asmussen (Asmussen (2000), Chapter VIII.4 , p. 229-233), and some results on Lévy processes with no negative jumps. 
Consider a risk process where the claims interarrival times are i.i.d with a general distribution function $F$, and the claim sizes are i.i.d independent of the arrival process phase-type distributed with representation $(\boldsymbol{\beta}, \mathbf{S})$, where $\mathbf{S}$ is an $n \times n$ square matrix. Let $u$ be the initial reserve, and let $\varphi(u)$ be the ruin probability. Then:

\section{Proposition 3.4.}

$$
\varphi(u)=\boldsymbol{\beta}^{+} e^{\left(\mathbf{S}+\mathbf{s} \boldsymbol{\beta}^{+}\right) u} \mathbf{1},
$$

where, $\mathbf{s}=-\mathbf{S 1}, \boldsymbol{\beta}^{+}$is the solution to the following equation:

$$
\boldsymbol{\beta}^{+}=\boldsymbol{\beta} \int_{0}^{\infty} e^{\left(\mathbf{S}+\mathbf{s} \boldsymbol{\beta}^{+}\right) y} F(d y),
$$

where $\mathbf{s} \boldsymbol{\beta}^{+}$is an $n \times n$ square matrix with $i, j$ element equal to $s_{i} \beta_{j}^{+}$.

(ii) Assume that there is a claim at time 0 with phase-type distribution with representation $\left(\boldsymbol{\beta}_{0}, \mathbf{S}\right)$, then the ruin probability is

$$
\varphi_{0}(u)=\boldsymbol{\beta}_{0} e^{\left(\mathbf{S}+\mathbf{s} \boldsymbol{\beta}^{+}\right) u} \mathbf{1}
$$

(iii) The deficit at ruin, given ruin occurs is phase-type with representation $(\boldsymbol{v}(u), \mathbf{S})$ in case (i), and $\left(\boldsymbol{v}_{0}(u), \mathbf{S}\right)$ in case (ii), where

$$
\begin{aligned}
\boldsymbol{v}(u) & =\frac{1}{\varphi(u)} \boldsymbol{\beta}^{+} e^{\left(\mathbf{S}+\mathbf{s} \boldsymbol{\beta}^{+}\right) u} \text { and }, \\
\boldsymbol{v}_{0}(u) & =\frac{1}{\varphi_{0}(u)} \boldsymbol{\beta}^{0} e^{\left(\mathbf{S}+\mathbf{s} \beta_{+}\right) u} .
\end{aligned}
$$

Proof. Statement (i) was proved by Asmussen ((Asmussen (2000)), Theorem 4.4, Chapter VIII, p. 230-231). Statements (ii) and (iii) follow from Corollary 2.3 and Theorem 4.4 in Chapter VIII, of Asmussen (2000).

The process $R_{1}$ is a compound Poisson risk process with phase-type claim amounts perturbed by a Brownian motion. Mordecki (2002) found the ruin probability for this model. His results are based on (Bertoin (1996)).

Let $Y(t)=-c t+\sigma B(t)$, where $B(t)$ is a Brownian motion, and let $\tilde{\psi}(z)=$ $-c z+\sigma^{2} z^{2} / 2$ be its Lévy exponent. Let $q_{\lambda}$ be the positive root of $\tilde{\psi}(z)=\lambda$. The following Proposition follows from Theorem 4, page 191, and Theorem 5, page 159, in Bertoin (1996).

Proposition 3.5. Let $\mathcal{M}(t)=\sup \{0 \leq s \leq t: Y(s)\}$. Let $E_{\lambda}$ be an exponentially distributed random variable independent of $Y(t)$, with parameter $\lambda$. Then

(i) $\mathcal{M}\left(E_{\lambda}\right)$ is exponentially distributed with parameter $q_{\lambda}$. 
(ii) Let $\theta>0$, and let $V=M\left(E_{\lambda}\right)-Y\left(E_{\lambda}\right)$, then

$$
\mathbb{E}(\exp \{-\theta V\})=\frac{\lambda}{\lambda-\tilde{\psi}(-\theta)} \frac{q_{\lambda}+\theta}{q_{\lambda}} .
$$

(iii) $V$ and $\mathcal{M}\left(E_{\lambda}\right)$ are independent.

Mordecki (2002) applied Proposition 3.5, and showed that the ruin probability for this model is the same as the ruin probability in a risk model, where the claims arrive according to a renewal process where the inter-arrival times are i.i.d distributed as $V$, and the claim sizes are i.i.d independent of inter-arrival times, with phase-type distribution with representation $((0, \boldsymbol{\beta}), \mathbf{S})$, where $\mathbf{S}$ is an $n+1 \times n+1$ matrix

$$
\mathbf{S}=\left(\begin{array}{cc}
q_{\lambda} & \mathbf{0} \\
\mathbf{t} & \mathbf{T}
\end{array}\right)
$$

The first arrival occurs at time 0 , with claim amount which is exponentially distributed with parameter $q_{\lambda}$. Let $\boldsymbol{\beta}^{+}$be as per (3.15) with $F=F_{V}$ being the distribution function of $V$. Proposition 3.4 implies that

1 .

$$
\varphi_{1}(b-a)=\mathbf{1}_{1}^{\prime} e^{\left(\mathbf{S}+\mathbf{s} \boldsymbol{\beta}^{+}\right)(b-a)} \mathbf{1},
$$

where $\mathbf{1}_{1}^{\prime}$ is an $n+1$ dimensional row vector with all components except the first, which is 1 .

2. The deficit at ruin for the process $R_{1}$, given that ruin occurs, is distributed as a random variable $\zeta_{1}$, which has a phase-type distribution with representation $\left(\left(v_{0}, v\right), \mathbf{S}\right)$, where

$$
\left(v_{0}, \boldsymbol{v}\right)=\frac{1}{\varphi_{1}(b-a)} \mathbf{1}_{1}^{\prime} e^{\left(\mathbf{S}+\mathbf{s} \boldsymbol{\beta}^{+}\right)(b-a)} .
$$

Note that $v_{0}$ is the atom at 0 .

To obtain the ruin probability in (3.14), we need to derive $\rho$ - the probability of reaching the level $b$ before ruin after the down-crossing the level $a$, and $\omega_{i}$, $i=0, \cdots, n$, the probability that ruin occurs due to diffusion or a claim in phase $i$. To obtain $\rho$, and $\omega_{i}$, we substitute $\mathbf{T}$ with $\mathbf{S}$, and $\boldsymbol{\eta}$ with $\boldsymbol{v}$ in equations (3.6)-(3.8).

Substituting $\rho$ and (3.19) in (3.14) we obtain the ruin probability.

The deficit at ruin given ruin occurs. Clearly the deficit at ruin given ruin occurs is phase-type with representation $\left(\left(\delta_{0}, \boldsymbol{\delta}\right), \mathbf{T}\right)$, where $\delta_{0}$ is the atom at 0 when ruin occurs due to diffusion. Let $\tilde{\delta}_{i}$ be the probability that ruin occurs at phase $i, i=0, \cdots, n$, then

$$
\tilde{\delta}_{i}=\gamma(u, i)+q(u) \omega_{i} \sum_{r=1}^{\infty} \rho^{r-1} \varphi_{1}(b-a)^{r}=\gamma(u, i)+\omega_{i} \frac{q(u) \varphi_{1}(b-a)}{1-\rho \varphi_{1}(b-a)} .
$$


Therefore the distribution of the deficit at ruin given ruin occurs is phase type with representation $\left(\left(\delta_{0}, \boldsymbol{\delta}\right), \mathbf{T}\right)$, where $\delta_{i}=\frac{\tilde{\delta}_{i}}{\varphi_{b}(u)}$.

\section{ACKNOWLEDGEMENT}

I want to thank the the Zimerman Foundation for the study of Banking and Finance for their financial support.

\section{REFERENCES}

Albrecher, H., Claramunt, M.M and Mármol, M. (2005) On the distribution of dividend payments in a Sparre Anderses model with generalizes Erlang(n) interclaim times. Insurance: Mathematics and Economics, 37, 324-334.

Asmussen, S., Nerman, O. and Olsson, M. (1996) Fitting phase-type distributions via EM algorithm. Scandinavian Journal of Statistics, 23, 419-441.

Asmussen, S. (2000) Marix-analytic models and their analysis. Scandinavian Journal of Statistics, 27, 193-226.

Asmussen, S. and Rolski T. (1991) Computational methods in risk theory: A matrix-algorithmic approach. Insurance: Mathematics and Economics, 10, 259-274.

Asmussen, S. (2000) Ruin Probabilities. World Scientific.

Asmussen, S. (2003) Applied probability and queues. Second edition. Springer.

Asmussen, S. and Kella, O. (2000) A multi-dimensional martingale for Markov additive processes and its applications. Advances in Applied Probability, 32, 376-393.

Bertoin, J. (1996) Lévy Processes. Cambridge university press.

Bratiychunk, M.S. and Derfla, D. (2007) On a modification of the classical risk process. Insurance: Mathematics and Economics, forthcoming.

Bühlmann, H. (1970) Mathematical Methods in Risk Theory. Springer-Verlag. Berlin Heidelberg New York.

De Finetti, B. (1957) Su un impostazione alternativa della teoria collectiva del rischio. Transactions of the XV International Congress of Actuaries 2, 433-443.

Dickson, D.C.M. and Waters, H.R. (2004) Some oprimal dividends problems. Astin Bulletin, 34, 49-74.

Frostig, E. (2005a) The expected time to ruin in a risk process with constant barrier via martingale. Insurance: Mathematics and Economics, 37, 116-228.

Frostig, E. (2005b) On the expected time to ruin and the expected dividends when dividends are paid while the surplus is above a constant barrier. Journal of Applied Probability, Vol. 42(3), 595-607.

Gerber, H.U. (1979) An Introduction to Mathematical Risk Theory. S.S. Hubner Foundation Monographs, University of Pennsylvania.

Gerber, H.U. and ShiU, E.S.W. (1998) On the time value of ruin. North American Actuarial Journal, 2(1), 48-78.

Gerber, H.U. and Shiu, E.S.W. (2004) Optimal dividends: Analysis with Brownian motion. North American Actuarial Journal, 8(1), 1-20.

Gerber, H.U. and Shiu, E.S.W. (2006) On optimal dividend strategies in the compound Poisson model. North American Actuarial Journal, 10, 76-93.

IRBÄCK, J. (2003) Asymptotic theory for a risk process with high dividend barrier. Scandinavian Actuarial Journal, 2, 97-118.

Kella, O. and WhitT, W. (1992) Useful martingales for stochastic storage processes with Lévy input. Journal of Applied Probability, 29, 396-403.

LI, S. and GARRIDO, J. (2004) On a class of renewal risk models with a constant duvidends barrier. Insurance: Mathematics and Economics, 35, 691-701. 
LI, S. (2006) The distribution of the dividend payments in the compound Poisson risk model perturbed by diffusion. Scandinavian Actuarial Journal, 2, 73-85.

Lin, S.X., Willmot, G. and Drekic, S. (2003) The classical risk model with constant dividend barrier: analysis of the Gerber-Shiu discounted penalty function. Insurance: Mathematics and Economics, 33, 551-566.

Lin, S.X. and Pavlova, K.P. (2006) The compound Poisson risk model with a threshhold dividend strategy. Insurance: Mathematics and Economics, 38, 57-80.

MordeCKI, E. (2002) The distribution of the maximum of a Lévy process with positive jumps of phase-type. Theory of Stochastic Processes, 8(24), 309-316.

Neuts, M.F. (1981) Matrix-geometric solusions in stochastic models. Johns Hopkins University Press, Baltimore.

Paulsen, J. and GJessing, H.K. (1997) Optimal choice of dividend barriers for a risk process with stochastic return on investments. Insurance: Mathematics and Economics, 20, 215-223.

ZHou, X. (2004) When does surplus reach a certain level before ruin? Insurance: Mathematics and Economics, 35, 553-561.

Zhou, X. (2005) On a classical risk model with a constant dividend barrier. North American Actuarial Journal, 9, 95-108.

ESTHER FROSTIG

Department of Statistics

University of Haifa

Haifa, Israel 